PREPARED FOR THE U.S. DEPARTMENT OF ENERGY, UNDER CONTRACT DE-AC02-76CH03073

PPPL-3933

PPPL-3933

UC-70

A Roadmap to Recent Developments in the Theory of Turbulent Generation of Long-wavelength Flows

by

John A. Krommes

March 2004

$\left.\stackrel{M}{M}\right|_{\substack{\text { PRInCETON PLASIMA } \\ \text { PHYSICS LABORATORY }}} ^{D}$

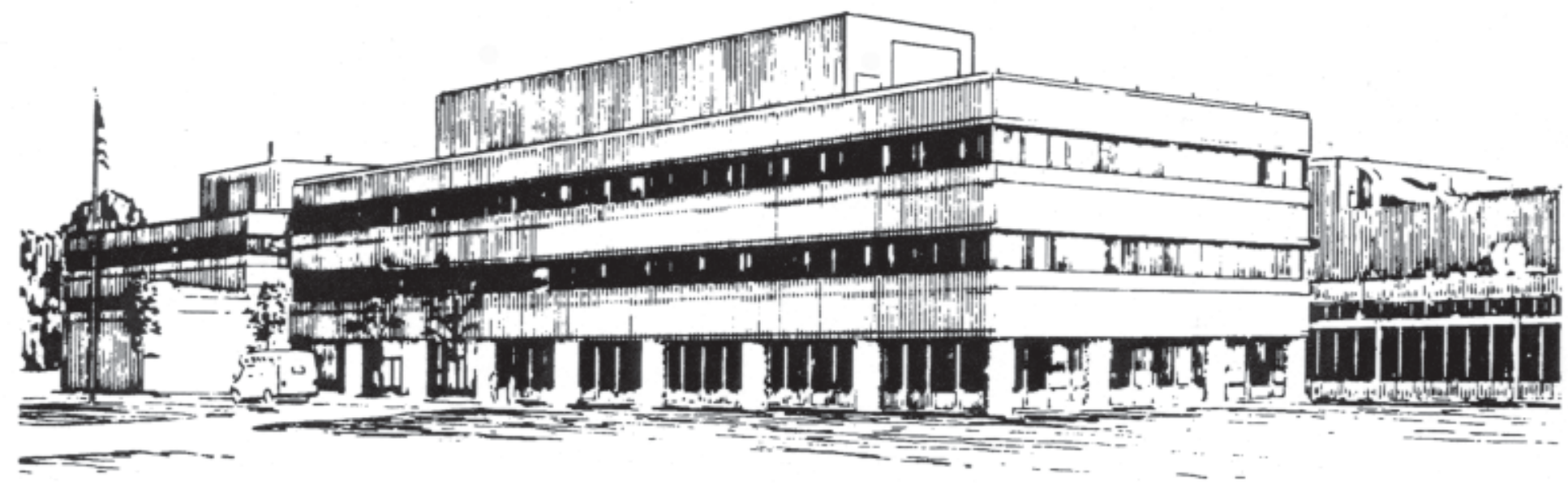

PRINCETON PLASMA PHYSICS LABORATORY PRINCETON UNIVERSITY, PRINCETON, NEW JERSEY 


\section{PPPL Reports Disclaimer}

This report was prepared as an account of work sponsored by an agency of the United States Government. Neither the United States Government nor any agency thereof, nor any of their employees, makes any warranty, express or implied, or assumes any legal liability or responsibility for the accuracy, completeness, or usefulness of any information, apparatus, product, or process disclosed, or represents that its use would not infringe privately owned rights. Reference herein to any specific commercial product, process, or service by trade name, trademark, manufacturer, or otherwise, does not necessarily constitute or imply its endorsement, recommendation, or favoring by the United States Government or any agency thereof. The views and opinions of authors expressed herein do not necessarily state or reflect those of the United States Government or any agency thereof.

\section{Availability}

This report is posted on the U.S. Department of Energy's Princeton Plasma Physics Laboratory Publications and Reports web site in Fiscal Year 2004. The home page for PPPL Reports and Publications is: http://www.pppl.gov/pub_report/

DOE and DOE Contractors can obtain copies of this report from:

U.S. Department of Energy

Office of Scientific and Technical Information

DOE Technical Information Services (DTIS)

P.O. Box 62

Oak Ridge, TN 37831

Telephone: (865) 576-8401

Fax: (865) 576-5728

Email: reports@adonis.osti.gov

This report is available to the general public from:

National Technical Information Service

U.S. Department of Commerce

5285 Port Royal Road

Springfield, VA 22161

Telephone: $1-800-553-6847$ or

(703) $605-6000$

Fax: (703) 321-8547

Internet: http://www.ntis.gov/ordering.htm 


\title{
A Roadmap to Recent Developments in the Theory of Turbulent Generation of Long-Wavelength Flows
}

\author{
KROMMES John A. \\ Plasma Physics Laboratory, Princeton University \\ P.O. Box 451, Princeton, NJ 08543 USA \\ e-mail:krommes@princeton.edu
}

\begin{abstract}
A brief overview is given of recent results in the theory of turbulent generation of long-wavelength fluctuations; references are provided to more detailed calculations in the literature. Emphasis is placed on conceptual foundations and technical tools, which can be simply illustrated by studies of the scalar (one-field) generalized Hasegawa-Mima equation. Then a functional Hamiltonian formalism is discussed as a way of efficiently handling systems of coupled fields. Casimir invariants are shown to be the appropriate "plasmon densities"; a tensor spectral-balance equation builds in the conservation constraints automatically. A concise expression can be obtained for the nonlinear growth rate in terms of the spectral density, a tensor generalization of the triad interaction time, and the metric tensor and structure constants of the Hamiltonian structure.
\end{abstract}

\section{Keywords:}

convective cell, zonal flow, drift wave, Hasegawa-Mima equation, EDQNM, Reynolds stress, eddy viscosity, Hamiltonian, Casimir invariant

\section{Introduction}

The theory of turbulent generation of long-wavelength flows is a topic of considerable current interest because of its connections to the general theory of drift-wave (DW) saturation and turbulent transport. In this paper I briefly discuss some of the recent developments in this area, including (i) the detailed study of the generalized Hasegawa-Mima equation (GHME) by Krommes and Kim (KK) [1]; (ii) the derivation of nonlinear energy theorems using field-theoretic techniques [1, 2]; and (iii) the Hamiltonian description of the generation process [2], which is especially well suited for treating systems of coupled partial differential equations but also yields further insights even for one-field theories. The emphasis is on conceptual foundations and technical tools; details can be found in the cited references. 
The specific calculations reported here have been conducted in slab geometry. They must not be applied blindly to toroidal situations [3], as there are important differences of both interpretation and detail (specifically, the wave number $k_{\|}$parallel to the magnetic field depends on the radial coordinate). With that caviat, I shall continue to use slab language for simplicity. I believe the technical tools are potentially useful quite generally, and various paradoxes and conceptual difficulties also have toroidal counterparts.

In slab geometry, by convective cell (CC) I shall mean any fluctuation (wavevector $\boldsymbol{q}$ ) with $q_{\|}=0$. That includes as special cases the particularly important zonal flows $\left(q_{y}=0\right.$; ZF's) as well as streamers $\left(q_{x}=0\right)$. Convective cells have highly nonadiabatic electron response [4]. One is concerned with the nonlinear generation rate $\gamma_{\boldsymbol{q}}$ of such fluctuations, which is one ingredient in the ultimate determination of the self-consistent turbulent steady state.

In understanding the role of ZF's and CC's in the saturation of DW turbulence, one general approach could be the development of nonlinear variational principles [5] that yield rigorous bounds on DW transport. Although preparatory groundwork has been performed [6-8] and an attempt has been made on the realistic problem of ion-temperature-gradientdriven turbulence [9], the theory is not yet in satisfactory shape. Here I will not discuss such bounding theories explicitly, but will rather focus on a different class of nonlinear energy principles for the $\mathrm{CC}$ growth rate. Those have intriguing implications for the more general problem.

A more technical motivation concerns the distinction between (i) the growth rate of modulational instability, and (ii) the nonlinear growth rate $\gamma_{\boldsymbol{q}}$ in a statistically steady state. Many authors have focused on modulational instability, but that assumes that DW's have already grown to a quasi-steady state while the CC's are not yet excited. This is valid only in an initial transient stage of evolution; in a statistically steady state, the techniques described in the subsequent sections must be employed.

\section{Statistical closure theory and the convective-cell growth rate}

Although robust Markovian closure theory has been well understood for some time (many references can be found in the recent review by Krommes [10]), a more heuristic analytical theory that focused specifically on ZF generation was initiated relatively recently by Diamond et al. [11]. That work was important and inspirational, but raised numerous questions concerning technical details (including the sign of the result), physical interpretations, and the relationship to more systematic closure theory. The subsequent work by Smolyakov and Diamond [12] on conserved quantities was also significant. Motivated by 
those works, KK [1] performed a detailed analysis of the GHME. They first discussed a direct asymptotic expansion of the eddy-damped quasinormal Markovian (EDQNM) formula [10] for nonlinear coherent damping (the turbulent generalization of collisional drag in classical Langevin theory), using the small parameter $\epsilon \doteq q / k \ll 1$, where $\boldsymbol{k}$ refers to the DW's. That calculation (surprisingly nontrivial in the general anisotropic case) has important links to the theory of negative eddy viscosity in 2D Navier-Stokes turbulence [13]; KK were able to make a quantitative connection. Because nonlinearity leads to interacting wavevector triads $\boldsymbol{k}+\boldsymbol{p}+\boldsymbol{q}=\mathbf{0}$, it is easily seen for fixed $\boldsymbol{k}$ and $|\boldsymbol{q}|$ that the basic interaction involves a DW $(\boldsymbol{k})$, two DW sidebands $\left(\boldsymbol{p}_{1}\right.$ and $\left.\boldsymbol{p}_{2}\right)$, and a $\mathrm{CC}(\boldsymbol{q}$ and $-\boldsymbol{q})$. However, the calculation is not of a modulational instability with fixed DW pump. Such analyses do not conserve energy between the DW's and CC's, which can lead to unforeseen and unphysical conclusions, including a spurious change of sign of the eddy viscosity (see the appendix of Ref. 1). The derivation from the EDQNM closure also shows that the appropriate "propagator" is the triad interaction time $\theta_{\boldsymbol{k}, \boldsymbol{k}, \boldsymbol{q}}$ between the modes $\boldsymbol{k},-\boldsymbol{k}$, and $\boldsymbol{q}$; this observation resolves a difficulty regarding overall sign and aids in the construction of an appropriate physical algorithm. Part of that algorithm involves consideration of weakly inhomogeneous spectral balance equations; KK also provided a reformulation and generalization of the results of Ref. 12 in terms of the standard Poisson-bracket formalism conventionally used in weakly inhomogeneous statistical dynamics, thereby resolving a paradox in the original theory [14]. One resulting insight is that it is unnecessary to formulate spectral balance equations in terms of an "action" or "plasmon density" invariant; the spectral density of any dependent variable can be used. That is particularly significant when $n$-field systems are considered, as discussed in Sec. 4 .

\section{Field theory and the modulation algorithm}

In Ref. 11 it was noted that $\gamma_{\boldsymbol{q}}$ was determined in terms of $\boldsymbol{q} \cdot \partial_{\boldsymbol{k}} \mathcal{Z}_{\boldsymbol{k}}$, where $\mathcal{Z}_{\boldsymbol{k}}$ was a certain quantity conserved by the DW's under the CC modulation. In the specific case of the GHME [1], $\mathcal{Z}_{\boldsymbol{k}}=\left(1+k^{2}\right)^{2}\left\langle\delta \varphi_{\boldsymbol{k}}^{2}\right\rangle$. It has been noted [12] that the expression for $\mathcal{Z}_{\boldsymbol{k}}$ is reproduced by the formula $\mathcal{Z}_{\boldsymbol{k}} \propto k_{y} E_{\boldsymbol{k}} / \omega_{\boldsymbol{k}}^{\mathrm{lin}}$, where $E_{\boldsymbol{k}} \doteq\left(1+k^{2}\right)\left\langle\delta \varphi_{\boldsymbol{k}}^{2}\right\rangle$ and $\omega_{\boldsymbol{k}}^{\text {lin }}$ is the DW frequency including polarization-drift corrections, namely $\omega_{k}^{\operatorname{lin}}=\omega_{*} /\left(1+k^{2}\right)\left(\omega_{*}\right.$ is the diamagnetic frequency). However, use of a linear frequency in this context is troubling because the $\mathrm{CC}$ generation process is nonlinear. Although linear physics is certainly relevant for determining the ultimate spectral properties of the saturated state, the work of KK showed clearly that the form of $\gamma_{\boldsymbol{q}}$ does not depend explicitly on the properties of linear waves; those enter only implicitly through the DW-CC triad interaction time 
and the ultimate form of the spectrum. In an attempt to better understand the foundations of the algorithm proposed in Ref. 11, KK used field-theory techniques to discuss the systematic derivation of a nonlinear energy principle for $\gamma_{\boldsymbol{q}}$. It is useful here to sketch the intuitive content of the formalism. Refer to Fig. 1 and assume that the system is statistically homogeneous and in steady state. (These restrictions are not fundamental.) Let $\psi$ denote the field variable(s). One expects that the $\mathrm{CC}$ growth rate $\gamma_{\boldsymbol{q}}$ is related to some sort of variation of the DW energy due to the presence of the CC's. Now the CC's randomly modulate the DW's; however, there is no mean field associated with the CC's because of the homogeneity. Furthermore, in a steady state the mean rate-of-change of the DW energy must vanish. Thus it is not the case that, for example, $\gamma_{\boldsymbol{q}} \propto-\left\langle\dot{\mathcal{E}}_{\mathrm{DW}}\right\rangle$. Clearly one must study the behavior of the DW fluctuations in the presence of the CC's, but one cannot look "too microscopically," e.g., consider $\left\langle\delta \widetilde{\mathcal{E}}_{\mathrm{DW}} / \delta \widetilde{\psi}_{\mathrm{CC}}\right\rangle$, since there will be cancellations due to the random phases of the turbulence. Rather, one needs to calculate the mean response function (Green's function) [10] for the turbulence. This observation [15] is a nonequilibrium generalization of the well-known fluctuation-dissipation theorem of equilibrium statistical mechanics, which relates the two-time correlation function $C$ of the random background fluctuations to the mean response function for infinitesimal perturbations away from that background. The technical solution that is now a standard tool of analytical statistical dynamics [15] is to break the symmetry by inserting an external source $\widehat{\eta}$. Now all statistical observables depend on $\widehat{\eta},\langle\psi\rangle_{\widehat{\eta}} \neq 0$, and two-point correlations (including energy spectra) can be considered to be functionally dependent on the induced mean field: $C=C[\langle\psi\rangle]$. This is illustrated in Fig. 2. It can be shown that $\gamma_{\boldsymbol{q}}$ is related to the first-order variation of the mean nonlinearity with respect to the induced mean field, calculated in the physical limit $\widehat{\eta}=0$. Technically, this result is related to the elimination of disconnected graphs that leads to the famous Dyson equation [16]. For much more information about such field-theoretic techniques, see Ref. 10.

Physically, the mean nonlinearity is just the Reynolds stress $\mathcal{S}$, and proper understanding of the method of sources just described is relevant to the interpretation of $\gamma_{\boldsymbol{q}}$ in terms of $\mathcal{S}$. Historically, $\mathcal{S}$ was defined to be the mean nonlinearity for a time-evolving flow in inhomogeneous geometry and is still used in that context in engineering-type modeling. In homogeneous turbulence $\mathcal{S}$ vanishes, but as discussed above the relevant quantity is not $\mathcal{S}$ itself but rather the variation of $\mathcal{S}$ due to an induced mean field. As an example, consider the Reynolds stress on CC's described by the GHME. Then $\mathcal{S}$ is the average of the nonlinear term describing advection of vorticity $\omega \doteq \nabla_{\perp}^{2} \varphi$. That average can be transformed [17] into an expression involving the Cartesian components of the $\boldsymbol{E} \times \boldsymbol{B}$ 
velocity (primes denote the DW's):

$$
\left.\left\langle\boldsymbol{V}_{E}^{\prime} \cdot \nabla \nabla^{2} \varphi^{\prime}\right\rangle=\left(\partial_{x x}-\partial_{y y}\right) \underline{\left\langle v_{x}^{\prime} v_{y}^{\prime}\right\rangle}+\partial_{x y} \underline{\left(\left\langle v_{y}^{\prime 2}\right\rangle-\left\langle v_{x}^{\prime 2}\right\rangle\right.}\right) .
$$

Now for homogeneous, isotropic turbulence each of the underlined terms vanishes by symmetry; there is no net Reynolds stress. But the situation is quite different when the DW turbulence is modulated by a CC with wavevector $\boldsymbol{q}$. Then neither of the underlined terms vanishes because the perturbed fluctuations are anisotropic (in a way dependent on $\boldsymbol{q}$ ). For arbitrary $\boldsymbol{q}$, both terms are of the same order and must both be retained in order to ensure a proper, rotationally covariant calculation of $\gamma_{\boldsymbol{q}}$. This argument is explained in more detail in Ref. 17.

\section{Hamiltonian description}

The CC growth rate $\gamma_{\boldsymbol{q}}$ is determined by nonlinear interactions. It is therefore useful to exploit the properties of the nonlinearities as much as possible. Seminal work by Morrison and Greene [18] showed that various Eulerian PDE's have a Hamiltonian structure; several decades of subsequent work was reviewed by Morrison [19]. I will show that the Hamiltonian formalism can be usefully applied to the calculation of $\gamma_{\boldsymbol{q}}$ and leads to an important new insight about the nature of the plasmon density. The methodology is particularly useful for systems of coupled PDE's, where one is led to very compact and elegant results.

In ordinary (noncanonical) Hamiltonian mechanics, the generalized coordinates evolve according to $\dot{z}^{i}=\left\{z^{i}, H\right\}$, where $H(z)$ is the Hamiltonian and $\{A, B\} \doteq\left(\partial_{z^{i}} A\right) J^{i j}\left(\partial_{z^{j}} B\right)$ (the antisymmetric matrix $\mathrm{J}$ being known as the Poisson tensor or the cosymplectic form). The use of noncanonical variables in plasma physics was pioneered by Littlejohn, exploited by Dubin et al. [20] in their noncanonical Hamiltonian formulation of the nonlinear gyrokinetic-Poisson system, and later generalized elegantly by Hahm [21] and others (for further references, see App. C of Ref. 10). When applied to an Eulerian PDE for a field $\psi(\boldsymbol{x}, t)$, an infinite-dimensional generalization is required $(i \rightarrow \boldsymbol{x})$. One may further generalize to a vector $\boldsymbol{\psi}$ of variables such as density or temperature. [I will also label those variables by an $i\left(\boldsymbol{\psi} \equiv \psi^{i}\right)$, although this $i$ should not be confused with the label of the original generalized coordinates.] One now requires a Hamiltonian functional $\mathcal{H}[\boldsymbol{\psi}]$ and an appropriate bracket $\{\cdot, \cdot\}$, which must satisfy antisymmetry $(\{A, B\}=-\{B, A\})$ and the Jacobi identity $(\{\{A, B\}, C\}+\{\{B, C\}, A\}+\{\{C, A\}, B\}=0)$. Then $\partial_{t} \boldsymbol{\psi}=\{\boldsymbol{\psi}, \mathcal{H}\}$. Motivated by the characteristic structure of the $\boldsymbol{E} \times \boldsymbol{B}$ advective nonlinearity, I define $[A, B] \doteq \widehat{\boldsymbol{z}} \cdot \boldsymbol{\nabla} A \times \boldsymbol{\nabla} B$. This "ordinary" bracket has the property $\overline{A[B, C]}=\overline{[A, B] C}$, 
where the overline denotes the integral over all space. I then choose

$$
\begin{gathered}
\mathcal{H}=\frac{1}{2} \overline{\psi^{i} g_{i j} \psi^{j}}=\frac{1}{2} \overline{\psi^{i} \psi_{i}}, \\
\{A, B\}=\overline{S^{r s}[\psi]\left[\frac{\delta A}{\delta \psi^{r}}, \frac{\delta B}{\delta \psi^{s}}\right] .}
\end{gathered}
$$

The symmetric matrix $g_{i j}$ is called the metric tensor and can be used to raise and lower indices, creating an appealing covariant formalism; note the covariant expression of $\mathcal{H}$ in Eq. (2). The symmetric matrix $S^{i j}$ is called the structure matrix and is taken to be linear in the fields: $S^{i j}[\boldsymbol{\psi}]=S^{i j}{ }_{k} \psi^{k}$. The $S^{i j}{ }_{k}$ are called the structure constants; they are constrained [2] in order that the Jacobi identity be satisfied. Using these formulas, one can demonstrate Hamiltonian structure for a number of the popular systems of PDE's of current interest [2], including, for example, the system of finite- $\beta$ collisional DW equations studied in Ref. 22.

It is easy to show that the bracket structure just defined conserves $\mathcal{H}$. Other quantities may be conserved as well. A Casimir invariant $\mathcal{C}$ is defined as any functional that is conserved without regard to the form of the Hamiltonian. That is, a Casimir is conserved purely because of the structure of the bracket. An example of a prospective Casimir is $\mathcal{C}=\frac{1}{2} \overline{\psi^{i} \psi^{i}}$. Then conservation of $\mathcal{H}$ and $\mathcal{C}$ can be proven very similarly by manipulating their time derivatives into contractions of symmetric and antisymmetric forms:

$$
\begin{aligned}
\partial_{t} \mathcal{H} & =-S^{i j}{ }_{k} \overline{\left[\psi^{k}, \psi_{j}\right] \psi_{i}}=-S^{i j}{ }_{k} \overline{\psi^{k}\left[\psi_{j}, \psi_{i}\right]}=0 \\
\partial_{t} \mathcal{C} & =-S^{i j}{ }_{k} \overline{\left[\psi^{k}, \psi_{j}\right] \psi_{i}}=-S^{i j}{ }_{k} \overline{\left[\psi^{i}, \psi^{k}\right] \psi_{j}}=0,
\end{aligned}
$$

the last result holding if $S^{i j}{ }_{k}=S^{k j}{ }_{i}$. Notice in particular how the proof of $\mathcal{C}$ conservation does not involve the covariant component $\psi_{j}$. Also note that this particular definition of $\mathcal{C}$ is not covariant in form, so a linear transformation of variables would change both its value and the specific symmetry property of the $S^{i j}{ }_{k}$ that ensures that $\mathcal{C}$ remains conserved. In general, it is a nontrivial task to identify all Casimir invariants of a given Hamiltonian structure.

Casimir conservation furnishes an important constraint on the dynamics, which are no longer allowed to explore the entire energy "surface". Consider in particular the interaction of DW's (denoted by primes) and CC's (denoted by underlines). Projecting the dynamics onto the DW subspace leads to

$$
\partial_{t} \psi^{i \prime}=-S^{i j}{ }_{k}(\underbrace{\left[\psi^{k \prime}, \underline{\psi}_{j}\right]}_{(\mathrm{a})}+\underbrace{\left[\psi^{k}, \psi_{j}^{\prime}\right]}_{(\mathrm{b})}+\underbrace{\left[\psi^{k \prime}, \psi_{j}^{\prime}\right]-\left[\psi^{k \prime}, \psi_{j}^{\prime}\right]}_{(\mathrm{c})}) .
$$


Actually, such a decomposition holds for any projection into two orthogonal subspaces, but it is particularly useful when a scale separation holds between the two classes of fluctuations. Term (a) describes the advection of DW's by CC's, term (b) describes the inverse process of CC's advected by DW's, and term (c) describes DW self-interactions. When the DW's are assumed to be of relatively short wavelength, term (b) is negligible, so term (a) describes the principal DW-CC interaction. Because $\underline{\psi}_{j}=\delta \mathcal{H} / \delta \underline{\psi}^{j}$ and the proof of Casimir conservation does not involve this covariant component of the field, this interaction conserves the DW Casimir(s). In particular, for the case of the GHME (which can easily be shown to be Hamiltonian), $\mathcal{Z}$ is a Casimir. This argument links the rather abstract concept of Casimir invariance with the quite physical process of nonlinear advection involving two disparate scales.

The same projection process can be exploited to derive a generalized Poynting theorem and nonlinear energy principle; for the details, see Ref. 2. The fundamental result is that if $\underline{\boldsymbol{Q}} \doteq\left\langle\underline{\psi^{i}}\right\rangle$ and $\underline{\boldsymbol{P}} \doteq\left\langle\underline{\psi}_{i}\right\rangle$, then second variations of the mean DW energy $\dot{\overline{\mathcal{E}}}_{\mathrm{DW}}$ with respect to $\underline{\boldsymbol{Q}}$ or $\underline{\boldsymbol{P}}$ produce co- or contravariant versions of the Hermitian part of the coherent damping tensor $\Sigma$, e.g.,

$$
\left(\underline{\boldsymbol{\Sigma}}_{q}^{H}\right)^{i j}=\frac{1}{2} \frac{\delta^{2} \dot{\overline{\mathcal{E}}}_{\mathrm{DW}}}{\delta \underline{P}_{\mathbf{q}, i}^{*} \delta \underline{P}_{\boldsymbol{q}, j}} .
$$

The scalar CC growth rate itself follows as a contraction of $\Sigma^{H}$ with the spectral tensor C according to $\gamma_{\boldsymbol{q}}=-\left[\left(\underline{\Sigma}_{q}^{H}\right)^{i j} \underline{C}_{j i}(\boldsymbol{q})\right] / \underline{C}_{k}^{k}(\boldsymbol{q})$.

The remaining manipulations involve only a modest, though detailed, amount of algebra: (i) write the tensor spectral-balance equation for $C_{\boldsymbol{k}}^{i j}$; (ii) obtain formulas for the nonlinear modulation frequencies in terms of the structure constants $S^{i j}{ }_{k}$ (one must be careful about co- and contravariant indices, which cannot be passed indiscriminately through Poisson brackets; the action of those $\boldsymbol{k}$-dependent brackets on the metric tensor is related to the energy loss of the DW's to the CC's due to wave-number refraction); (iii) write the equation for $\dot{\overline{\mathcal{E}}}_{\mathrm{DW}}=\frac{1}{2} \partial_{t}\left(C_{i}^{i}\right)_{\mathrm{DW}}$; (iv) carry out the required functional derivatives; (v) insert an appropriate triad interaction tensor $\widehat{\boldsymbol{\theta}}$; (vi) manipulate to obtain the final formula [2], which except for contractions over tensor indices is identical in form with the result of the scalar calculations [1]. I stress that in this derivation one does not need to guess or even be inspired about the form of a possibly preferred plasmon density; conservation of the proper Casimir(s) is built in. The elegant and concise way in which the formalism deals with those invariants provides a powerful argument in favor of further applications of the Hamiltonian methodology.

In the $n$-field formalism, the triad interaction time generalizes to a fourth-rank tensor $\widehat{\boldsymbol{\theta}} \equiv \theta^{i j}{ }_{k l}$, which must not be treated cavalierly. An important preliminary exercise is to 
demonstrate that a nontrivial $n$-field model (such as the electrostatic Hasegawa-Wakatani equations [23]) reduces correctly in the adiabatic DW limit to the GHME result; this is not obvious because the tensor theory couches $\gamma_{\boldsymbol{q}}$ in terms of $\boldsymbol{q} \cdot \partial_{\boldsymbol{k}} C_{\boldsymbol{k}}^{i j}$, whereas $\mathcal{Z}_{\boldsymbol{k}}$ is a $k$-dependent weighted contraction of $C_{\boldsymbol{k}}^{i j}$. The resolution is that $\widehat{\boldsymbol{\theta}}$ cannot be assumed to be diagonal. In the adiabatic limit it can be shown to approach a singular matrix times the scalar $\theta_{\boldsymbol{k},-\boldsymbol{k}, \boldsymbol{q}}$; this nontrivial matrix structure is one way in which the physics of linear theory enters the formula for $\gamma_{\boldsymbol{q}}$.

\section{Summary and conclusions}

Explicit statistical calculations on the generalized Hasegawa-Mima equation, including systematic asymptotic reduction of Markovian closure theory, application of the method of sources to the derivation of nonlinear energy principles, and proper derivation of weakly inhomogeneous spectral balance equations, have led to deeper understanding of the asymptotic problem defined by the nonlinear interactions of long-wavelength convective cells with short-wavelength drift waves. Further application of an infinite-dimensional Hamiltonian formalism shows that the DW's conserve Casimir invariants under the CC modulation. For multiple fields, the nonlinear CC generation process is constrained by the conservation of possibly multiple Casimirs, but a succinct formula can be obtained [2] in which those constraints are neatly built in. Applications of that formula are in progress [24]. It is hoped that the conceptual ideas and technical tools discussed here will stimulate further research into both toroidal generalizations and robust variational descriptions of steady-state microturbulence.

\section{Acknowledgements}

In various phases of this work I enjoyed collaborations with C.-B. Kim and R. A. Kolesnikov. I am grateful for informative discussions with B. Scott, T.-S. Hahm, and G. Hammett. This research was supported by U.S. Department of Energy Contract No. DE-AC02-76CHO-3073.

\section{References}

[1] J. A. Krommes and C.-B. Kim, Phys. Rev. E 62, 8508 (2000).

[2] J. A. Krommes and R. A. Kolesnikov, Hamiltonian description of zonal-flow generation, Phys. Plasmas (2003) (in press). 
[3] L. Chen, Z. Lin, and R. White, Phys. Plasmas 7, 3129 (2000).

[4] G. W. Hammett, M. A. Beer, W. Dorland, S. C. Cowley, and S. A. Smith, Plasma Phys. Control. Fusion 35, 973 (1993).

[5] F. H. Busse, Adv. Appl. Mech. 18, 77 (1978).

[6] J. A. Krommes and R. A. Smith, Ann. Phys. (N.Y.) 177, 246 (1987).

[7] C.-B. Kim and J. A. Krommes, J. Stat. Phys. 53, 1103 (1988).

[8] C.-B. Kim and J. A. Krommes, Phys. Rev. A 42, 7487 (1990).

[9] C.-B. Kim and K.-O. Choi, Phys. Plasmas 3, 4463 (1996).

[10] J. A. Krommes, Phys. Rep. 360, 1 (2002).

[11] P. H. Diamond, M. N. Rosenbluth, F. L. Hinton, M. Malkov, J. Fleischer, and A. Smolyakov, 17th IAEA Fusion Energy Conference (International Atomic Energy Agency, Vienna, 1998), p. 1421, IAEA-CN-69/TH3/1.

[12] A. I. Smolyakov and P. H. Diamond, Phys. Plasmas 6, 4410 (1999).

[13] R. H. Kraichnan, J. Atmos. Sci. 33, 1521 (1976).

[14] G. F. Carnevale and P. C. Martin, Geophys. Astrophys. Fluid Dynamics 20, 131 (1982).

[15] P. C. Martin, E. D. Siggia, and H. A. Rose, Phys. Rev. A 8, 423 (1973).

[16] F. J. Dyson, Phys. Rev. 75, 486 (1949).

[17] J. A. Krommes, Comments on "Dynamics of zonal flow saturation in strong collisionless drift wave turbulence" [Phys. Plasmas 9, 4530 (2002)], Phys. Plasmas (2003) (in press).

[18] P. J. Morrison and J. M. Greene, Phys. Rev. Lett. 45, 790 (1980).

[19] P. J. Morrison, Rev. Mod. Phys. 70, 467 (1998).

[20] D. H. E. Dubin, J. A. Krommes, C. R. Oberman, and W. W. Lee, Phys. Fluids 26, 3524 (1983).

[21] T. S. Hahm, Phys. Fluids 31, 2670 (1988). 
[22] S. J. Camargo, B. D. Scott, and D. Biskamp, Phys. Plasmas 3, 3912 (1996).

[23] A. Hasegawa and M. Wakatani, Phys. Rev. Lett. 50, 682 (1983).

[24] R. A. Kolesnikov and J. A. Krommes, Bull. Am. Phys. Soc. 48(7), 73 (2003). 


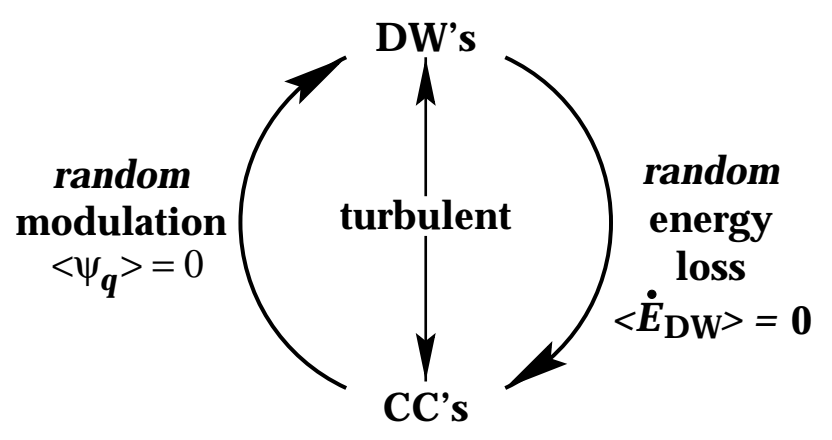

Figure 1: Modulation and energy transfer in homogeneous, steady-state turbulence. All mean fields vanish and there is no net energy transfer between the DW's and CC's. 


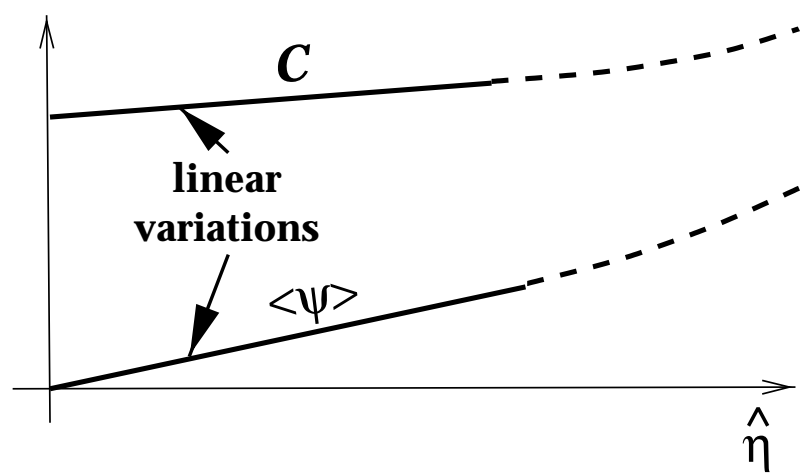

Figure 2: As $\widehat{\eta}$ is varied, the symmetry of the physical state $\widehat{\eta}=0$ is broken, $\langle\psi\rangle_{\hat{\eta}} \neq 0$, and $C=C[\langle\psi\rangle]$. 


\section{External Distribution}

Plasma Research Laboratory, Australian National University, Australia

Professor I.R. Jones, Flinders University, Australia

Professor João Canalle, Instituto de Fisica DEQ/IF - UERJ, Brazil

Mr. Gerson O. Ludwig, Instituto Nacional de Pesquisas, Brazil

Dr. P.H. Sakanaka, Instituto Fisica, Brazil

The Librarian, Culham Laboratory, England

Mrs. S.A. Hutchinson, JET Library, England

Professor M.N. Bussac, Ecole Polytechnique, France

Librarian, Max-Planck-Institut für Plasmaphysik, Germany

Jolan Moldvai, Reports Library, Hungarian Academy of Sciences, Central Research Institute for Physics, Hungary

Dr. P. Kaw, Institute for Plasma Research, India

Ms. P.J. Pathak, Librarian, Institute for Plasma Research, India

Ms. Clelia De Palo, Associazione EURATOM-ENEA, Italy

Dr. G. Grosso, Instituto di Fisica del Plasma, Italy

Librarian, Naka Fusion Research Establishment, JAERI, Japan

Library, Laboratory for Complex Energy Processes, Institute for Advanced Study, Kyoto University, Japan

Research Information Center, National Institute for Fusion Science, Japan

Dr. O. Mitarai, Kyushu Tokai University, Japan

Dr. Jiangang Li, Institute of Plasma Physics, Chinese Academy of Sciences, People's Republic of China

Professor Yuping Huo, School of Physical Science and Technology, People's Republic of China

Library, Academia Sinica, Institute of Plasma Physics, People's Republic of China

Librarian, Institute of Physics, Chinese Academy of Sciences, People's Republic of China

Dr. S. Mirnov, TRINITI, Troitsk, Russian Federation, Russia

Dr. V.S. Strelkov, Kurchatov Institute, Russian Federation, Russia

Professor Peter Lukac, Katedra Fyziky Plazmy MFF UK, Mlynska dolina F-2, Komenskeho Univerzita, SK-842 15 Bratislava, Slovakia

Dr. G.S. Lee, Korea Basic Science Institute, South Korea

Institute for Plasma Research, University of Maryland, USA

Librarian, Fusion Energy Division, Oak Ridge National Laboratory, USA

Librarian, Institute of Fusion Studies, University of Texas, USA

Librarian, Magnetic Fusion Program, Lawrence Livermore National Laboratory, USA

Library, General Atomics, USA

Plasma Physics Group, Fusion Energy Research Program, University of California at San Diego, USA

Plasma Physics Library, Columbia University, USA

Alkesh Punjabi, Center for Fusion Research and Training, Hampton University, USA

Dr. W.M. Stacey, Fusion Research Center, Georgia Institute of Technology, USA

Dr. John Willis, U.S. Department of Energy, Office of Fusion Energy Sciences, USA

Mr. Paul H. Wright, Indianapolis, Indiana, USA 
The Princeton Plasma Physics Laboratory is operated by Princeton University under contract with the U.S. Department of Energy.

\author{
Information Services \\ Princeton Plasma Physics Laboratory \\ P.O. Box 451 \\ Princeton, NJ 08543
}

Phone: 609-243-2750

Fax: 609-243-2751

e-mail: pppl_info@pppl.gov

Internet Address: http://www.pppl.gov 